\title{
Impacto del Plan de Atención Básica en la Prevención de Leishmaniasis Cutánea en Zonas Rurales de Santander, Colombia
}

\author{
Laura A. Rodríguez-Villamizar ${ }^{1}$, Luis C. Orozco- Vargas ${ }^{2}$ Y Gerardo Muñoz-Mantilla ${ }^{3}$ \\ ${ }^{1}$ Médica. Departamento de Salud Pública, Facultad de Salud. Universidad Industrial de Santander. \\ E-mail: laurarovi@guajiros.udea.edu.co \\ ${ }^{2}$ Médico. M. Sc. Escuela de Enfermería-Centro de Investigaciones Epidemiológicas. Universidad \\ Industrial de Santander. E-mail: lcorovar@uis.edu.co \\ ${ }^{3}$ Bacteriólogo. PhD. Escuela de Medicina, Departamento de Ciencias Básicas. Centro de Investigaciones \\ Epidemiológicas. Universidad Industrial de Santander. E-mail: germun@uis.edu.co
}

Recibido15 Noviembre 2005/Enviado para Modificación 1 Marzo 2006/Aceptado 12 Abril 2006

\section{RESUMEN}

Objetivo Evaluar el impacto de los programas del Plan de Atención Básica en la prevención de leishmaniasis cutánea en tres municipios santandereanos.

Materiales y Métodos Estudio ecológico con unidad de análisis vivienda. Se seleccionaron aleatoriamente niños menores de diez años de las escuelas rurales, se aplicaron encuestas estructuradas por vivienda y se examinó la presencia de cicatriz/lesión. Se hizo un análisis de regresión binomial ajustando por conglomerados.

Resultados Se muestrearon un total de 18 veredas con 284 viviendas y 609 niños menores de diez años. La prevalencia general de niños con cicatriz/lesión fue de 19,1 \% distribuida en forma diferencial en los tres municipios. El 42,8 \% de las viviendas reportaron haber recibido algún tipo de información y el 39,9 \% algún tipo de intervención de leishmaniasis del Plan de Atención Básica. Las intervenciones del Plan de Atención Básica mostraron un efecto protector en el análisis bivariado, pero luego de ajustar por variables socioeconómicas y ambientales, su efecto no fue significativo (PR=1,39 IC95 \% 0,76-2,56)

Conclusión Las intervenciones del Plan de Atención Básica en leishmaniasis no tienen asociación significativa con la presencia de cicatriz/lesión en los niños menores de diez años, probablemente porque su efecto es superado por las condiciones socioeconómicas y ambientales de la zona, así como por las condiciones administrativas relacionadas con la baja cobertura de los programas. 
Palabras Clave: Leishmaniasis cutánea, evaluación, salud pública, Colombia (fuente: DeCS, BIREME).

\section{ABSTRACT}

The Basic Health Plan's impact on preventing cutaneous leishmaniasis in rural areas of Santander, Colombia

Objective Measuring the impact of the Basic Colombian Health Plan for Preventing Cutaneous Leishmaniasis in three towns in the Santander department.

Methods An ecologic design using housing as the analysis unit. Children aged less than ten were randomly selected from rural schools and visited in their homes by trained volunteers. Structured survey-forms were filled in and every child was examined for scars or active cutaneous leishmaniasis lesions. Cluster-adjusted binomial regression was used when analysing data.

Results Eighteen villages, 284 houses and 609 children aged less than 10 were surveyed. Scar/lesion prevalence was 19,1\%, differences being noted between municipalities: El Playón 7,1 \%, Landázuri 32,8 \% and Rionegro $13,9 \%$. Information regarding leishmaniasis had been received by $42,8 \%$ of the houses and $39,9 \%$ had received some type of intervention from the $\mathrm{Ba}$ sic Colombian Health Plan, bed-nets being the most frequent (92,5\%). Basic Health Plan intervention showed a preventative effect in bivariate analysis; however, such effect was not evident following adjustment for other socioeconomic and environmental variables (PR=1,39 IC95 \% 0,76-2,56).

Conclusion Basic health plan intervention for cutaneous leishmaniasis prevention has had no significant association with scar/lesion prevalence in children aged less than ten in these rural areas, probably because its effect has been exceeded by local socioeconomic and environmental conditions, as well as administrative conditions related to the programmes' poor coverage.

Key Words: cutaneous leishmaniasis, evaluation, public health, Colombia (source: MeSH, NLM).

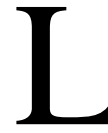

as Leishmaniasis son un grupo de enfermedades parasitarias distribuidas mundialmente con múltiples manifestaciones clínicas y causadas por una variedad de especies de protozoos pertenecientes al género Leishmania que son transmitidas en América por la picadura de flebótomos hembra pertenecientes al género Lutzomyia $(1,2)$. En Latinoamérica la enfermedad es endémica reportándose prevalencias que varían entre los países pero que en general son mayores del $20 \%$ en las zonas rurales medida como reactividad a la prueba cutánea $(3,4)$. 
En Colombia, la leishmaniasis se presenta en forma endémica en focos naturales de infección en zonas rurales siendo notificados anualmente en el país alrededor de 8500 casos (5). En Santander, durante el 2004 se reportaron 900 casos de leishmaniasis cutánea para una tasa de 44 casos por 100 000 habitantes, la segunda más alta después del dengue dentro de las enfermedades de notificación obligatoria. La tasa de incidencia más alta se encontró en el municipio de Landázuri con 2357 casos por 100000 habitantes. En El Playón y Rionegro las tasas de incidencia fueron de 420 y 103 casos de leishmaniasis por 100000 habitantes, respectivamente (6).

En el municipio de Landázuri, uno de los principales focos endémicos de Santander, el estudio de Muñoz en 1996 (7) estableció por medio de prueba cutánea (que correlacionó muy bien con presencia de cicatrices y lesiones activas), una prevalencia acumulada en población general de 75 \% identificando que entre los niños de diez años la prevalencia se situaba alrededor del 60 \%. Estos datos apoyan la hipótesis epidemiológica que, de manera similar a lo reportado en otros países, la transmisión en estas áreas de Santander ocurre primordialmente en la periferia y el interior de las viviendas (5,8-11).

Las estrategias de control en leishmaniasis son de tipo ambiental o grupal y se centran en los vectores y reservorios (12). En Colombia, éstas intervenciones dirigidas a la prevención de enfermedades que afectan a la colectividad, están a cargo del estado por medio del Plan de Atención Básica (PAB), cuya reglamentación y obligatoriedad está establecida en el territorio nacional por la resolución 4288 de 1996.

Los estudios de evaluación han permitido mejorar la eficiencia y efectividad de los programas en enfermedades transmitidas por vectores (ETV), especialmente en África (13). El objetivo de este estudio es evaluar el impacto de los programas del PAB en la prevención de leishmaniasis y determinar los factores asociados con la presencia de cicatriz/lesión cutánea en tres municipios santandereanos donde la enfermedad se considera endémica.

\section{MATERIALES Y MÉTODOS}

Población de estudio

El estudio se realizó en los municipios de El Playón, Landázuri y Rionegro en el departamento de Santander (Colombia) dada su alta incidencia de Leishmaniasis y la cooperación de sus dirigentes y comunidad con el proyecto (6). Los tres municipios son de predominio rural y tienen una tempe- 
ratura aproximada de 25 grados centígrados, una precipitación anual que varía entre 1500 y 2000 milímetros y una humedad relativa promedio de 80 $\%$. La actividad económica predominante es la agricultura, especialmente de productos como cacao y café.

Diseño del estudio y tamaño de muestra

Se utilizó un diseño de tipo ecológico de comparación de grupos múltiples tomando como unidad de análisis las viviendas $(14,15)$. Se calculó en el programa Stata 8.0 un tamaño de muestra mínimo de 257 viviendas teniendo en cuenta una prevalencia de infección en niños de diez años de $60 \%$, un cambio mínimo del $10 \%$, un poder $90 \%$ y un nivel de confianza de $95 \%$.

Recolección de datos y diagnóstico de Leishmaniasis

Se seleccionaron un mínimo de cinco veredas por municipio para ser incluidas en el estudio. Posteriormente, del censo de estudiantes rurales de básica primaria de cada vereda se seleccionaron con tabla de números aleatorios, 20 niños nativos y menores de diez años (o el número de niños de estas características si era menor a 20). Se tomaron como individuos de estudio sólo los menores de diez años debido a que el PAB tiene sólo este tiempo de vigencia como plan de beneficios colectivo. Debido a que la escolaridad en zona rural se inicia en promedio a los seis años de edad, para incluir en el estudio niños entre 0 y 6 años se muestrearon las viviendas de los niños seleccionados y se incluyeron en el estudio todos los menores de diez años de la vivienda, nativos y residentes en la vereda. Se escogieron cinco viviendas con presencia de niños no escolarizados en cada vereda para controlar el sesgo de selección debido a que la cobertura escolar es del $80 \%$. Por cada vivienda incluida en el estudio se seleccionaron a los padres de familia o adulto responsable mayor de 18 años para contestar los datos relacionados con la vivienda y el entorno domiciliario. Los encuestadores fueron agentes de salud voluntarios de cada vereda que han venido siendo preparados en la prevención y control de leishmaniasis desde hace aproximadamente dos años y que tienen amplia experiencia en la presentación clínica de la forma cutánea de la enfermedad. El diagnóstico de presencia de cicatriz o lesión activa de leishmaniasis en los menores de diez años de las viviendas muestreadas fue dado por estos agentes de salud voluntarios teniendo en cuenta las características típicas de este tipo de lesión: úlceras redondeadas de bordes levantados o cicatrices deprimidas redondeadas (16).

Variables de estudio 
La variable de salida fue la presencia cicatriz/lesión en los menores de diez años. Como variable explicatoria principal se tomó la presencia de acciones del PAB definidas como: haber recibido información/educación o haber recibido intervenciones de barrera específicas (fumigación, toldillos o collares para caninos). Con el fin de ajustar el efecto del PAB por el efecto de otras variables asociadas en la literatura con la presencia de cicatriz/lesión se recogieron las siguientes variables adicionales: ubicación de la casa a menos de 150 metros de plantaciones de cacao o café, distancia en metros a la casa vecina, materiales de los pisos, paredes y techos, conocimiento de la enfermedad, reconocimiento del vector, conocimiento de atención gratuita, conocimiento acerca del papel del perro doméstico en la transmisión de la enfermedad, presencia de caninos en la vivienda, presencia de caninos enfermos en la vivienda, uso de fumigación intradomiciliaria, uso de toldillo en los dormitorios, percepción subjetiva de la acción de la junta de acción comunal, percepción subjetiva del estado de las vías de acceso, tiempo y dinero empleado hasta la cabecera municipal. Como variables específicas de los niños muestreados se recogieron los datos de edad y sexo $(11,17,18)$.

Manejo y análisis de datos

Se crearon bases de datos independientes para los datos provenientes de la vivienda/entorno y los datos específicos de los niños (edad, sexo, presencia de cicatriz o lesión activa). Se trabajaron los datos conjuntos como bases relacionales en el programa EpiInfo 6.04d y se llevaron a una sola base de datos definitiva para ser analizada en el programa Stata 8.0. En el análisis descriptivo de la población de estudio se utilizaron medidas de posición central y dispersión para variables cuantitativas y proporciones para variables discretas. Para el análisis bivariado y estratificado se tuvieron en cuenta las posibles variables confusoras del impacto del PAB, determinando su efecto crudo y ajustado en la presencia de cicatriz/lesión en los niños. Se tomó como unidad de análisis la vivienda debido a las características de transmisión de leishmaniasis cutánea en la zona. Se utilizó un modelo de regresión binomial para el análisis multivariado. Posteriormente, con el fin de determinar la bondad de ajuste estadística y gráfica del modelo final, éste se analizó con una regresión logística para encuestas teniendo como unidad de análisis las viviendas y como estrato el municipio (19).

\section{Consideraciones éticas}

El estudio tuvo en cuenta las normas éticas vigentes en Colombia acordes con la Declaración de Helsinki. Se solicitó consentimiento informado escrito a los padres o adultos responsables y se obtuvo el asentimiento de los niños 
y niñas. Los menores con lesiones activas fueron remitidos para recibir tratamiento en el hospital local.

\section{RESULTADOS}

\section{Características de la población}

Se muestrearon un total de 18 veredas con 284 viviendas y 609 niños menores de diez años. Las características de las viviendas se presentan en la Tabla 1. En la constitución de las casas predomina el uso de techo de zinc en los tres municipios; sin embargo, se aprecian diferencias marcadas en el material de sus paredes y pisos. El promedio de niños menores de diez años por casa fue de dos con un rango entre uno y seis niños, sin diferencias por sexo. Existen dificultades de acceso a las veredas muestreadas que se evidencian por el estado regular de las vías de acceso y el tiempo empleado hasta la cabecera municipal que tuvo una mediana de dos horas (rango 0-360 minutos).

Tabla 1. Características de las viviendas según municipio, zona rural Santander, 2004

\begin{tabular}{|c|c|c|c|c|c|c|c|c|}
\hline \multirow[t]{2}{*}{ Variable / Municipio } & \multicolumn{2}{|c|}{ El Playón } & \multicolumn{2}{|c|}{ Landázuri } & \multicolumn{2}{|c|}{ Rionegro } & \multicolumn{2}{|c|}{ Total } \\
\hline & No. & $\%$ & No. & $\%$ & No. & $\%$ & No. & $\%$ \\
\hline $\begin{array}{l}\text { Ubicación menor a } 150 \\
\text { metros de plantaciones }\end{array}$ & 64 & 86,5 & 77 & 74,8 & 88 & 84,6 & 229 & 81,5 \\
\hline $\begin{array}{c}\text { Materiales del techo } \\
\text { Zinc }\end{array}$ & 74 & 97,4 & 99 & 96,2 & 73 & 70,2 & 246 & 86,9 \\
\hline $\begin{array}{l}\text { Otros (teja, cielo raso) } \\
\text { Materiales del piso }\end{array}$ & 2 & 2,6 & 4 & 3,8 & 31 & 29,8 & 37 & 13,1 \\
\hline Tierra & 52 & 68,4 & 12 & 11,6 & 45 & 43,7 & 109 & 38,5 \\
\hline Cemento & 24 & 31,6 & 36 & 34,6 & 48 & 56,3 & 118 & 41,7 \\
\hline Madera (tabla) & 0 & 0 & 56 & 53,8 & 0 & 0 & 56 & 19,8 \\
\hline Materiales de las paredes & & & & & & & & \\
\hline Bahareque o tapia & 62 & 81,5 & 0 & 0 & 31 & 30,2 & 93 & 32,9 \\
\hline Ladrillo & 10 & 13,2 & 14 & 13,6 & 60 & 58,2 & 84 & 29,8 \\
\hline Madera (tabla) & 4 & 5,3 & 89 & 86,4 & 12 & 11,6 & 105 & 37,3 \\
\hline $\begin{array}{l}\text { Distancia casa vecina en } \\
\text { metros }\end{array}$ & & & & & & & & \\
\hline Mediana - Rango & 350 & & 200 & & 200 & & 200 & $(0-6000)$ \\
\hline
\end{tabular}

Prevalencia de cicatriz o lesión de leishmaniasis

La prevalencia general de niños con cicatriz/lesión fue de 19,1 \% distribuida en forma diferencial en los municipios: El Playón 7,1 \%, Landázuri 32,8 \% y Rionegro 13,9 \%. Teniendo en cuenta las viviendas con presencia de al menos un niño afectado por leishmaniasis la prevalencia general fue de $27,2 \%$. En el análisis bivariado se encontró un aumento de riesgo de $10 \%$ para la presencia de cicatriz/lesión por cada año de edad adicional. En la distribu- 
ción de la presencia de cicatriz/lesión por edades de acuerdo con el uso de toldillo en el dormitorio, se observó una tendencia lineal creciente más pronunciada para los niños que no lo usan.

Cobertura del plan de atención básica

El 42,8 \% de las viviendas reportaron haber recibido algún tipo de información de Leishmaniasis provenientes del PAB municipal o departamental. De la misma manera, el 39,9 \% ha recibido algún tipo de intervención siendo mayoritario el suministro de toldillos (92,5 \%) frente a la fumigación (7,5 $\%)$. La correlación entre las variables de información e intervención del PAB fue buena con un rho de Spearman y estadística phi de 59,3 \%, dado principalmente por la correlación en el municipio de Rionegro, pero muy baja para los otros dos municipios (menos del $18 \%$ ). Se creó una variable única de salida denominada pab definida como haber recibido cualquiera de las dos intervenciones obteniendo una prevalencia general del $51 \%$ distribuida en forma diferencial en los municipios: El Playón 65,8 \%, Landázuri 5,8 \% y Rionegro 85,3 \%.

\section{Factores relacionados con presencia de cicatriz}

Las variables que en forma independiente mostraron asociación con la presencia de cicatriz/lesión fueron: PAB, ubicación cercana a cultivos de cacao o café, viviendas con suelo y paredes de madera, el no uso de toldillo y fumigación intradomiciliaria, la edad, la presencia de perros en la vivienda, el conocimiento de la enfermedad, el conocimiento de la gratuidad de la atención ,tiempo mayor de dos horas hasta la cabecera municipal y costo del traslado mayor a \$6 000. El análisis del efecto crudo del PAB se hizo por separado para las actividades de información (PR crudo de 0,68 IC95 \% 0,470,99), intervención con barreras (PR crudo de 0,66 IC95 \% 0,46-0,96) y para la variable unificada (PR crudo de 0,56 IC95 \% 0,39-0,80). De este análisis preliminar se destaca el aparente efecto protector de las intervenciones PAB.

\section{Impacto del PAB en Leishmaniasis cutánea}

Los resultados del modelo multivariado final se muestran en la Tabla 2. En este análisis se muestra que luego de ajustar el efecto de las intervenciones del $\mathrm{PAB}$ por otras variables asociadas con la presencia de cicatriz/lesión se pierde su efecto protector haciéndose no significativo. De esta forma, el tipo de construcción de la vivienda, representado por el material de sus paredes; el conocimiento de la enfermedad por parte de los adultos; la presencia de perros en la vivienda; el costo del traslado desde la vereda hasta la cabecera 
municipal y la edad del niño fueron las variables que mostraron tener mayores efectos sobre la presencia de cicatriz o lesión activa en los menores de diez años en estas zonas rurales. Las pruebas estadísticas y gráficas evidencian que no hay pérdida del ajuste o inestabilidad del modelo, producido por la presencia de observaciones extremas (Test de 10 grupos de Hosmer-Lemeshow $\mathrm{p}=0,87$ ).

\section{DISCUSIÓN}

El trabajo se realizó sólo en tres municipios santandereanos, que a pesar de ser pocos representan el espectro de enfermedad en la zona rural y se encuentran dentro de los más afectados en el departamento (6). Los encuestadores fueron residentes voluntarios, que teniendo sólo una experiencia previa como encuestadores, registraron en forma completa y adecuada la información. Reportes preliminares de la validación de su concepto diagnóstico ha encontrado valores de sensibildad de $90 \%$ y especificidad de $83 \%$ con respecto al diagnóstico parasitológico.

Tabla 2. Análisis multivariado de los factores asociados a la presencia de cicatriz/lesión de leishmaniasis en menores de diez años, zona rural Santander

\begin{tabular}{|c|c|c|c|c|}
\hline Variable & $\begin{array}{c}\text { Prevalencia } \\
\text { relativa }\end{array}$ & $\begin{array}{l}\text { Error estándar } \\
\text { semirobusto }\end{array}$ & $\begin{array}{c}\text { Valor } \\
\mathrm{p}\end{array}$ & $1 C^{a} 95 \%$ \\
\hline Intervención PAB barreras $^{b}$ & 1,39 & 0,4320 & 0,288 & $0,76-2,56$ \\
\hline Intervención PAB información & 1,02 & 0,2698 & 0,950 & $0,60-1,71$ \\
\hline Vivienda con paredes de tabla & 2,47 & 0,5975 & 0,000 & $1,59-3,96$ \\
\hline Edad & 1,11 & 0,0308 & 0,000 & $1,05-1,17$ \\
\hline $\begin{array}{l}\text { Conocimiento de la enfermedad } \\
\text { por padres/cuidadores }\end{array}$ & 2,65 & 0,9022 & 0,004 & $1,35-5,16$ \\
\hline Presencia de perros en vivienda & 2,02 & 0,6939 & 0,042 & $1,02-3,95$ \\
\hline Costo traslado mayor a $\$ 6.000^{c}$ & 1,82 & 0,4086 & 0,007 & $1,18-2,83$ \\
\hline
\end{tabular}

Intervalo de Confianza

Incluye uso de toldillos y fumigación

${ }^{c}$ Costo de traslado desde la vereda hasta la cabecera municipal

Se encontró que el acceso a los programas de control de leishmaniasis es inequitativo en las zonas de difícil acceso y que en estos lugares la prevalencia de cicatriz/lesión en los menores de diez años ha permanecido casi constante después de la inclusión del PAB como plan de beneficios colectivo.

La zona rural estudiada es cacaotera por excelencia, factor que ha sido asociado a la presencia de la enfermedad (20), pero que en este estudio carece de asociación, posiblemente debido a que los tres municipios comparten esta condición. En concordancia con otros estudios como los realizados en Tumaco, Nariño $(11,21,22)$ en éstas zonas también se demuestra que las per- 
sonas que residen en viviendas construidas con paredes de tabla tienen mayor riesgo de adquirir la enfermedad, comparadas con residentes en viviendas de bloques de cemento o ladrillo; éste indicador puede reflejar de alguna manera las condiciones socioeconómicas de la zona (y de los municipios).

El conocimiento de la enfermedad por parte de los padres o cuidadores de los niños es otra variable que demostró una asociación fuerte en el modelo multivariado, pero que debe ser interpretada con cuidado, pues es posible que su asociación este dada porque las personas que manifiestan conocer la leishmaniasis sean las personas que han tenido contacto con adultos o niños enfermos.

La presencia de perros en la vivienda ha sido asociada, en este trabajo y en estudios previos, con la presencia de enfermedad y se explica por el patrón documentado de transmisión peridomiciliaria en esta zona (7), relacionado no sólo con el cambio de hábitat del vector sino también con la condición de reservorio de los caninos $(22,23)$.

La distancia desde las veredas hasta la cabecera municipal es un factor que dificulta el diagnóstico y el tratamiento oportuno de las personas y que puede generar inequidad dentro de la comunidad por la mayor posibilidad de los adultos para desplazarse (24). De igual forma las zonas más alejadas de la zona urbana, por la dificultad logística que representan, pueden tener menos posibilidad de recibir intervenciones del PAB.

La prevalencia de cicatriz/lesión en los niños de diez años en Landázuri para 1996 era de 60 \% con una media geométrica de la edad en los casos incidentes con síntomas clínicos de 3,8 años y de enfermedad subclínica de 8,7 años (7). En este nuevo estudio, la prevalencia en los niños de siete años fue de $60,7 \%$, evidenciando que no existen cambios en un período de ocho años. Aunque la prevalencia general ha disminuido (32,8 \%), la relación de la presencia de cicatriz/lesión con la edad sigue representando un riesgo acumulativo que es casi dos veces mayor en los niños mayores de cinco años comparados con los menores; ésta diferencia refleja la mayor probabilidad de infección por un mayor tiempo de exposición a la transmisión intradomiciliaria, aunque también puede relacionarse con la mayor movilidad de los niños mayores de cinco años por el inicio de la vida escolar y por lo tanto, con una mayor exposición al vector.

En el análisis preliminar, la relación de la edad con la presencia de cicatriz mostró ser modificada por el uso de toldillos en las viviendas, siendo consistente con reportes de diferentes países, especialmente africanos (25- 
27), donde la estrategia de uso de toldillos impregnados con permetrina ha demostrado un impacto positivo en la morbilidad y mortalidad de los niños debido a enfermedades transmitidas por vectores, como leishmaniasis. Sin embargo, en el análisis multivariado ésta relación no demostró significancia. Dos posibles explicaciones para este hallazgo pueden ser: primero, que en las zonas estudiadas los toldillos tienen menos cobertura precisamente en la zonas de mayor transmisión y segundo, el hecho de que la medición de uso del toldillo se hizo para la vivienda pero no específicamente para cada uno de los niños participantes, sesgo que ocurrió de forma no diferencial y que puede llevar a la nulidad la fuerza de la asociación entre las dos variables. En este sentido, consideramos que la relación del uso de toldillos con la presencia de infección/cicatriz debe ser analizada con más detalles en este estudio y en estudios posteriores.

La evaluación de los programas de salud pública ha sido una herramienta gerencial fundamental para el mejoramiento de los mismos y comprende la evaluación integral de los procesos de planeación, implementación, así como de sus resultados e impacto (28). En Colombia, desde la implementación de la reforma, su evaluación ha sido extensa en el plan de beneficios individual $(29,30)$ pero escasa para el plan colectivo (PAB) y se ha centrado más en los procesos administrativos y de implementación. Recientemente fue publicada una evaluación administrativa del PAB en 212 municipios descentralizados de Colombia, donde se concluye que la construcción de una autonomía territorial para asumir la salud y la organización incipiente de los municipios y de las direcciones locales de salud, influyeron en el desarrollo de los lineamientos del PAB, estando éste plan por debajo de las necesidades de salud pública de los colombianos (31).

Como indicador de proceso, éste estudio permite mostrar que la cobertura general de acciones del PAB en estas zonas santandereanas de alto riesgo de transmisión del leishmaniasis no alcanza el $50 \%$ y es menor del $5 \%$ en Landázuri, que constituye la zona de transmisión más activa; este hallazgo posiblemente esté relacionado con el hecho de que este municipio está más alejado de la capital departamental y presenta mayores dificultades de acceso.

Como evaluación de impacto, hasta nuestro conocimiento ésta es la primera evaluación que se realiza para un área específica de acción del PAB en Colombia. En este campo, el presente estudio aporta el conocimiento de que existe un efecto protector crudo del PAB, y que éste es similar para las intervenciones de información y las acciones de barrera contra el vector; sin embargo, éste efecto no tiene asociación significativa con la presencia de cica- 
triz/lesión en los niños menores de diez años, pues su resultado es superado por la fuerza de las condiciones socioeconómicas y ambientales de la zona, así como las condiciones administrativas relacionadas con la inequidad en la cobertura de los programas. De esta forma, el replanteamiento de los programas de prevención y control de la leishmaniasis en estas zonas deben orientarse a la ampliación de la cobertura a regiones de difícil acceso y el mejoramiento de las condiciones sociales y de vivienda de estas zonas rurales.

Finalmente, el presente estudio representa un acercamiento desde los métodos epidemiológicos a la evaluación de los planes de beneficios del actual sistema de salud en Colombia con la participación de la comunidad •

Agradecimientos. A los trabajadores sociales comunitarios y a todos los voluntarios de las veredas participantes, por su trabajo arduo y desinteresado en beneficio de la comunidad. Trabajo cofinanciado por COLCIENCIAS (código 11020412926) y la Universidad Industrial de Santander.

\section{REFERENCIAS}

1. Desjeux P. Human leishmaniases: epidemiology and public health aspects. Health Stat Q 1992;45:267-75.

2. Desjeux P. Leishmaniasis. Public health aspects and control. Clin Dermatol 1996:14:417-24

3. Marrano N, Mata L, Durack D. Cutaneous leishmaniasis in rural Costa Rica. Trans R Soc Trop Med Hyg 1989;83:340.

4. Maingon R, Feliciangeli D, Guzmán B, Rodríguez N, Conviti J, Adamson R, et al. Cutaneous leishmaniasis in Táchira state, Venezuela. Ann Trop Med Parasitol 1994;88:29-36.

5. Vélez I, Hendrickx E, Robledo S, Agudelo S. Leishmaniosis cutánea en Colombia y género. Cad Saude Publica 220;17:171-80.

6. Observatorio de Salud Pública de Santander, Organización Panamericana de la Salud. Situación de salud en Santander. Indicadores básicos 2005. Bucaramanga; 2005.

7. Muñoz G, Davies C. Leishmania panamensis transmission in the domestic environment: the results of a prospective epidemiological survey in Santander, Colombia. Biomédica, Volumen 25, en prensa.

8. Jones T, Johnson W, Barreto A, Lago E, Badaro R, Cerf B, et al. Epidemiology of american cutaneous leishmaniasis due to leishmania braziliensis braziliensis. J Infect Dis 1987;156:73-83.

9. Armijos R, Weigel M, Izurieta R, Racines J, Zurita C, Herrera W, et al. The epidemiology of cutaneous leishmaniasis in sutropical ecuador. Trop Med Int Health 1997;2:140-52. 
10. Passos V, Falcao A, Marzochi C, Gontijo C, Dias E, Barbosa-Santos E, et al. Epidemiological aspects of american coutaneous leishmaniasis in a peruvian area of the metropolian region of Belo Horizonte, Minas Gerais, Brazil. Mem Inst Oswaldo Cruz 1993;88:103-10.

11. Yadon Z, Rodriguez L, Davies C, Quingle M. Indoor and peridomestic transmission of american cutaneous leishmaniasis in northwestern argentina: a retrospective case-control study. Am J Trop Med Hyg 2003;68:519-26.

12. Davies C, Caye P, Croft S, Sundar S. Leishmaniasis. New approach to disease control. BMJ 2003;326:377-82.

13. Bryce J, Roungou J, Nguyen-Dinh P, Naimoli J, Breman J. Evaluation of nacional malaria control programmes in Africa. Bull World Health Organ1994;72:371-81.

14. Morgenstern H. Uses of ecologic analysis in epidemiologic research. Am J Public Health 1982;72:1336-44.

15. Borja-Aburto VH. Estudios ecológicos. Salud Publica Mex 2000;42:533-8.

16. Weigle C, Gore S. Natural History, clinical evolution, adn the host-parasite interaction in the new world cutaneous Leishmaniasis. Clin Dermatol 1996;14:433-50.

${ }^{17}$. Alcais A, Abel L, David C, Torrez M, Flandre P, Dedet J. Risk factors for onset of cutaneous and mucocutaneous leishmaniasis in Bolivia. Am J Trop Med Hyg 1997;57:79-84.

18. Weigle K, Santrich C, Martínez F, Valderrama L, Saravia N. Epidemiology of cutaneous leishmaniasis in Colombia: enviromental and behavoral risk factors for infection, clinical manifestations, and pathogenicity. J Infect Dis 1993;168:709-14.

19. Hosmer DW, Lemeshow S. Applied logisitc regression. New York: John Wiley \& Sons; 1989.

20. Scorza J, Valera M, Moreno E, Jaimes R. Encuesta epidemiológica sobre la lesihmaniasis cutánea, un estudio en Mérida, Venezuela. Bol Oficina Sanit Panam 1983;95:118-31.

21. Armijos R, Weigel M, Izurieta R, Racines J, Zurita C, Herrera W, et al. The epidemiology of cutaneous leishmaniasis in sutropical ecuador. Trop Med Int Health 1997;2:140-52.

22. Rojas C. An ecosystem approach to human health and the prevention of cutaneous leishmaniasis in Tumaco, Colombia. Cad Saude Pública 2001; 17 suppl:193-200.

23. Reithinger R, Davies C. Is the domestic dog (canis familiares) a reservoir host of american cutaneous leishmaniasis? A critical review of the current evidence. Am J Trop Med Hyg 1999;61:530-41.

24. Vélez I, Hendrickx E, Robledo S, Agudelo S. Leishmaniosis cutánea en Colombia y género. Cad Saude Publica 220;17:171-80.

25. Kroeger A, Villegas E, Morison L. Insecticida impregnated curtains to control domestic transmisión of cutaneous leishmaniasis in Venezuela: cluster randomised trial. BMJ 2002;325:810-3.

26. Ter K, Terlouw D, Philips-Howard P, Hawley W, Friedman J, Kolczak M, et al. Impact of permethrin-treated bed net son malaria an all-case morbidity in 
young children in an area of intense perennial malaria transmision in western kenya: crosssectional survey. Am J Trop Med Hyg 2003;68 suppl: 1007.

27. Diallo DA, Cousens S, Cuzin-Ouattara N, Ilboudo-Sanogo E, Esposito F. Chile mortality in west african population protected with insecticida-treated curtains for a period up to 6 years. Bull World Health Organ 2004;82:85-91.

28. Habicht JP, Victoria CG, Vaugham JP. Evaluation designs for adecuacy, plausibility and probability of public health programme performance and impact. Int J Epidemiology 1999;28:10-8.

29. Cardona A, Nieto E, Arbeláez MP, Agudelo H, Chávez B, Montoya A, Estrada A. Impacto de la reforma de seguridad social sobre la organización institucional y la prestación de los servicios de salud en Colombia. Facultad Nacional de Salud Pública, Medellín; 1999.

30. Bello A, Vega R. El sistema colombiano de seguridad social en salud: desigualdad y exclusión. Revista gerencia y políticas de salud 2001;1:48-73.

31. Chávez M, Sierra O, Lopera M, Montoya P. El plan de atención básica en los municipios descentralizados de Colombia, 2001-2003. Revista Facultad Nacional de Salud Pública 2004;22:77-89. 